\title{
Noncompact Lagrangian Manifolds Corresponding to the Spectral Series of the Schrödinger Operator with Delta-Potential on a Surface of Revolution
}

\author{
T. Ratiu ${ }^{a}$, T. A. Filatova ${ }^{b}$, and A. I. Shafarevich ${ }^{c}$ \\ Presented by Academician A.T. Fomenko April 9, 2012
}

Received April 16, 2012

DOI: $10.1134 / \mathrm{S} 1064562412050365$

Let $M$ be a Riemannian manifold, and let $V: M \rightarrow \mathbb{R}$ be a smooth function (potential). It is well known (see, e.g., [5]) that if the Hamiltonian system in $T^{*} M$ determined by the Hamiltonian $\frac{1}{2}|p|^{2}+V$ is completely integrable, then the corresponding Liouville tori $\Lambda$ determine the quasiclassical spectral series of the operator $H=-\frac{h^{2}}{2} \Delta+V(x)$ (here, $x \in M$, $(x, p)$ denotes the standard coordinates on $T^{*} M$, and $h>0$ ). Namely, the asymptotics as $h \rightarrow 0$ of the eigenvalues of the operator $H$ is calculated from the Bohr-Sommerfeld-Maslov quantization conditions

$$
\frac{1}{2 \pi h} \int_{\gamma}(p, d x)+\frac{1}{4} \mu(\gamma)=m \in \mathbb{Z},
$$

where $\gamma$ is an arbitrary cycle on $\Lambda, \mu$ is the Maslov index, and $m=O(1 / h)$. The formal asymptotics of the eigenfunctions (quasi-modes) has the form $\psi=K_{\Lambda}(1)$, where $K_{\Lambda}$ is the Maslov canonical operator on the torus $\Lambda$ satisfying the quantization condition.

Schrödinger operators with point potentials (known also as zero-radius potentials, or delta-potentials) are used in many mathematical and physical problems (see, e.g., [1-3]): on the one hand, such operators have interesting spectral properties, and on the other hand, thanks to the point support of the potential, they can be effectively studied in many cases. The spectral theory of operators with delta-

\footnotetext{
a Polytechnic Federal School of Lausanne, Station 8 CH-1015 Lausanne, Switzerland e-mail: tudor.ratiu@epfl.ch

${ }^{b}$ Moscow Institute of Physics and Technology, Institutskii per. 9, Dolgoprudnyi, Moscow oblast, 141700, Russia

${ }^{c}$ Mechanics and Mathematics Faculty, Moscow State University, Moscow, 119991 Russia e-mail: shafarev@yahoo.com
}

potentials has been extensively developed in the past decade; at the same time, the geometry of the corresponding classical problem has been little studied.

This paper describes Lagrangian manifolds determining the spectral series of the Schrödinger operator with delta-potential on a 2-surface of revolution diffeomorphic to the sphere. These manifolds are invariant with respect to the geodesic flow, but, unlike the Liouville tori, noncompact; the asymptotics of the eigenvalues of the Schrödinger operator is calculated from nonstandard quantization conditions on them. This paper is a continuation of paper [10], in which the case of the standard sphere was considered; in this case, unlike in the situation described below, the spectrum of the Schrödinger operator can be calculated exactly. The questions of integer-valuedness, which are related to invariants of geodesics flows, were discussed earlier in $[7,8]$.

\section{STATEMENT OF THE PROBLEM}

The Schrödinger operator

$$
H=-\frac{h^{2}}{2} \Delta+\alpha \delta\left(x-x_{0}\right), \quad \alpha \in \mathbb{R},
$$

on a compact 2-surface of revolution diffeomorphic to the sphere is determined on the basis of two natural considerations. First, it is required that this operator be self-adjoint; secondly, at the functions vanishing at the point $x_{0} \in M$, this operator must coincide with the usual Laplacian. These two requirements lead to the following formal definition. Let $\Delta$ be the LaplaceBeltrami operator on $M$; the domain of this operator is the second Sobolev space $H^{2}(M)$. We denote the restriction of $\Delta$ to the subspace of functions vanishing at the point $x_{0}$ by $H_{0}$; this operator is symmetric on $L^{2}(M)$.

Definition 1. The Schrödinger operator with deltapotential is a self-adjoint extension $H$ of the symmetric operator $H_{0}$. 
Remark 1. All extensions are parameterized by one parameter $\alpha$, which can be naturally interpreted as the coefficient of the delta-function (at $\alpha=0$, we obtain the Laplace-Beltrami operator).

Remark 2. The domain of the operator $H$ consists of functions having singularities at the point $x_{0}$; to be more precise, these functions have the form

$$
f\left(x, x_{0}\right)=-\frac{a(f)}{2 \pi} \ln r\left(x, x_{0}\right)+b(f)+o(1),
$$

and

$$
a(f)=\frac{2 \alpha}{h^{2}} b(f),
$$

where $a(f), b(f) \in \mathbb{C}$ and $r\left(x, x_{0}\right)$ is the distance between the points $x$ and $x_{0}$ on $M$.

In what follows, it is assumed that $x_{0}$ is a pole of the surface of revolution; our purpose in this paper is to describe the eigenvalue series of the operator $H$ in the quasiclassical limit as $h \rightarrow 0$; main attention is paid to constructing the corresponding Lagrangian manifolds. The surface $M$ is assumed to be analytic; to be more precise, we assume that $M$ is given in $\mathbb{R}^{3}$ by the parametric equations

$$
x=(f(z) \cos \varphi, f(z) \sin \varphi, z),
$$

where $f(z)$ is an analytic function in some neighborhood of the interval $\left(\left(z_{1}, z_{0}\right)\right)$ in the complex plane of the variable $z$. The points $x_{0}=\left(z_{0}, \varphi\right)$ and $x_{1}=\left(z_{1}, \varphi\right)$ are poles of the surface of revolution $M$; in their neighborhoods, the following conditions hold $f\left(z_{0}\right)=f\left(z_{1}\right)=0$ and, as $z \rightarrow z_{0}$ and $z \rightarrow z_{1}$,

$$
f(z)=\sqrt{z_{0}-z w(z)}, \quad f(z)=\sqrt{z_{1}-z \vee(z)},
$$

where $w(z), v(z)$ are analytic functions near the points $z=z_{0}$ and $z=z_{1}$, respectively.

\section{STATEMENT OF THE RESULT}

In the cotangent space $T_{x_{0}}^{*} M$, consider the circle $\Lambda_{0}: x=x_{0},|p|=2 E, E=$ const. The 2-manifold $\Lambda_{1}=\bigcup_{t} g_{t} \Lambda_{0}$, where $g_{t}$ is the geodesic flow on $M$, is diffeomorphic to the torus, and the noncompact manifold $\Lambda=\Lambda_{1} \backslash \Lambda_{0}$ is diffeomorphic to the infinite cylinder.

It is easy to prove the following assertion.

Statement 1. For any $E$, on the cylinder $\Lambda$, the Bohr-Sommerfeld-Maslov quantization condition holds, and there exists a Maslov canonical operator $K_{\Lambda}: C^{\infty}(\Lambda) \rightarrow C^{\infty}(M)$ with volume form $d s \wedge d t$, where $s$ is the natural parameter on the circle $\Lambda_{0}$ and $t$ is time on the orbits of the geodesic flow.
The main result of this paper is the following theorem.

Theorem 1. Suppose that there exists a number $E=$ $O(1)$ satisfying the quantization condition

$$
\tan \left(\frac{1}{2 h} \oint_{\gamma}(p, d x)+O(h)\right)=\frac{2}{\pi}\left(\ln \left(\frac{\sqrt{2 E}}{h}\right)+\frac{\pi h^{2}}{\alpha}+c\right),
$$

where $\gamma$ is any orbit of the geodesic flow on the Lagrangian manifold $\Lambda$ and $c$ is the Euler constant.

Then there exists an eigenvalue $E_{0}$ of the operator $H$ for which $\left|E-E_{0}\right|=o(h)$ as $\eta \rightarrow 0$. The corresponding eigenfunction has the form

$$
\psi=K_{\Lambda}(1)+O(h) .
$$

Remark 3. The quantization condition is a condition on the cohomology class of the cylinder $\Lambda$ corresponding to the homology with closed supports (each homology group is generated by the orbit of the geodesic flow going "from infinity to infinity" on the cylinder).

Remark 4. As $\left(\frac{\pi h^{2}}{\alpha}+\ln \frac{1}{h}\right) \rightarrow \infty$, the quantization condition takes the standard form

$$
\oint_{\gamma}(p, d x)=2 \pi h\left(k+\frac{1}{2}\right),
$$

where $k \in \mathbb{R}_{+}\left(k \sim \frac{1}{h}, h \rightarrow 0\right)$.

If the right-hand side in the quantization condition tends to a constant $B$ as $h \rightarrow 0$ (this happens at $\alpha=\frac{\pi h^{2}}{B+\ln h}+o(1)$, then), then the equation for the spectrum takes the form

$$
\tan \left(\frac{1}{2 h} \oint_{\gamma}(p, d x)\right)=\frac{1}{\pi}(\ln 2 E+2 c+B) .
$$

Note that the similar distinguished case $\alpha=O\left(h^{2} / \ln h\right)$ arises in the study of the convergence of the spectrum of an operator with smooth delta-like potential to the spectrum of an operator with delta-potential in the Euclidean plane [1].

\section{MONODROMY OF AN EQUATION WITH REGULAR SINGULAR POINTS}

The proof of the theorem is based on studying the monodromy of the equation to which is the spectral problem for the operator $H$ reduces after the separation of variables. There are two regular singular points corresponding to the poles of the surface $M$ in this equation, and both monodromy operators related to these points are equivalent to Jordan cells. The eigenfunctions of the operator $H$ correspond special solutions of the equation; namely, for the monodromy operator related to the point $x_{1}$, the solution must determine an eigenvector, and for the operator corresponding to the point $x_{0}$, it must determine a fixed associated vector. The asymptotic for such a solution is 
constructed in several steps. First, by using the complex WKB method developed by Fedoryuk [9], the monodromy operator corresponding to the point $x_{1}$ is calculated and the asymptotics of its eigenvector is determined. Then, by using the Langer-Wasow method [4], the asymptotics of the required associated vector of the second monodromy operator is described. At the last step, the local solutions are glued together into a global solution; at this step, topological condition (1) arises.

\section{ACKNOWLEDGMENTS}

This work was supported by the state program for support of research headed by leading scientists at the federal state government-financed higher educational institution "Moscow State University" (contract no. 11 G.34.31.0054).

\section{REFERENCES}

1. S. Albeverio, F. Gesztesy, R. Høegh-Krohn, and H. Holden, Solvable Models in Quantum Mechanics (Am. Math. Soc., Providence, R.I., 2005).
2. S. Albeverio and P. Kurasov, Singular Perturbations of Differential Operators (Cambridge Univ. Press, Cambridge, 2000).

3. F. A. Berezin and L. D. Faddeev, Dokl. Akad. Nauk SSSR 137, 1011-1014 (1961).

4. W. R. Wasow, Asymptotic Expansions for Ordinary Differential Equations (Wiley, New York, 1965; Mir, Moscow, 1968).

5. V. P. Maslov, Asymptotic Methods and Perturbation Theory (Nauka, Moscow, 1988) [in Russian].

6. V. P. Maslov and M. V. Fedoryuk, Quasi-Classical Approximation for Equations of Quantum Mechanics (Nauka, Moscow, 1976) [in Russian].

7. A. T. Fomenko, Usp. Mat. Nauk 44 (1), 145-173 (1989).

8. A. T. Fomenko, Izv. Akad. Nauk SSSR, Ser. Mat. 50 (6), 1276-1307 (1986).

9. M. V. Fedoryuk, Asymptotic Methods for Linear Ordinary Differential Equations (Nauka, Moscow, 1977) [in Russian].

10. T. A. Filatova and A. I. Shafarevich, Teor. Mat. Fiz. 164 (2), 279-298 (2010). 